\title{
How colour palettes in maps are re-coloured by daltonization methods
}

\author{
Anne Kristin Kvitle ${ }^{\mathrm{a}, *}$ \\ ${ }^{a}$ The Norwegian Universal Design Research Laboratory, Department of Manufacturing and Civil Engineering, NTNU \\ Norwegian University of Science and Technology, anne.kvitle@ntnu.no \\ * Corresponding author
}

Keywords: accessible maps, colour vision deficiency, re-colouring, daltonization

\begin{abstract}
:
The ability of identifying objects and elements based on colour is important in order to decode the information in a map or other information graphics. For this reason, the colours need to appear correct and be perceived in the desired and intended way. Map reading is reported as a challenging task for people with impaired colour vision. In reviews of the challenges of colour vision deficiencies (CVD) in everyday life (Cole, 2004), up to $60 \%$ of the subjects in the studies reported problems in reading colour coded charts, slides and prints. Other studies (Carter and Silverstein, 2010) describes the difficulties to distinguish and identify coloured objects in weather, financial and other maps and charts.
\end{abstract}

Colour vision deficiencies are common, where congenital CVD affects about $8 \%$ of the male population and $0.4 \%$ of the female population. In addition, colour vision and colour perception may be affected by medical conditions or injury (acquired CVD) and situational conditions (situation induced CVD).

Reviews of visual usability and accessible map design conclude that few maps appear to have been designed with CVD users in mind (Cartwright, 2015) and that the design efforts or research of accessible colours palettes for CVD observers are mostly limited to thematic maps such as choropleths (Kvitle, 2018).

Daltonization methods are image processing methods to automatically enhance information in existing images. A common enhancement method is re-colouring, changing the colours in the original image to make be more distinguishable to the CVD observers. The daltonization method targets a specific type of CVD, and may also have been designed for specific applications (natural images, scientific images, information graphics etc). Therefore, the evaluation of the methods is often based on a limited set of test images. Using one specific map image as input will give very different results based on the daltonization methods.

The aim of the work is primarily to examine how the colour palettes in a map are altered by different daltonization methods. Second, the aim is to explore how different map types are influenced by the daltonization methods and to propose requirements and guidelines for test images for future work.

The set of test images in this work includes

- Information graphics (such as a tube map).

- Choropleth map.

- Reference map based on different map providers.

To illustrate the visual differences, CVD simulation methods are applied on the original images and the daltonized versions of the images.

\section{References:}

Carter, R. C., \& Silverstein, L. D. (2010). Size matters: Improved color-difference estimation for small visual targets. Journal of the Society for Information Display, 18(1), 17-28. doi:10.1889/JSID18.1.17.

Cartwright, W. (2015). Assessing Cartographic Products for Visual Usability. In J. Brus, A. Vondrakova, \& V. Vozenilek (Eds.), Modern Trends in Cartography: Selected Papers of CARTOCON 2014 (pp. 269-279). Cham: Springer International Publishing. 
Cole, B. L. (2004). The handicap of abnormal colour vision. Clinical and Experimental Optometry, 87(4-5), $258-275$.

Kröger, J., Schiewe, J., \& Weninger, B. (2013). Analysis and improvement of the open-streetmap street color scheme for users with color vision deficiencies. Paper presented at the Proceedings of the 26th international cartographic conference, Dresden.

Kvitle, A.K. (2018). Accessible maps for the color vision deficient observers: past and present knowledge and future possibilities. Proceedings of the ICA, Volume 1. 